\title{
Perfil dos alunos e conhecimentos produzidos no Programa de Pós-Graduação de Enfermagem na Saúde do Adulto
}

\author{
STUDENTS PROFILE AND ACADEMIC PRODUCTION OF THE ADULT \\ HEALTH NURSING GRADUATE PROGRAM \\ PERFIL DE LOS ALUMNOS Y CONOCIMIENTOS PRODUCIDOS EN EL PROGRAMADE
POSTGRADO DE ENFERMERÍA EN SALUD DELADULTO
}

\section{Ruth Natalia Teresa Turrini ${ }^{1}$, Kazuko Uchikawa Graziano ${ }^{2}$, Maria de Fátima Fernandes Vattimo ${ }^{3}$, Diná de Almeida Lopes Monteiro da Cruz ${ }^{4}$}

\author{
1 Professor Doutor do \\ Departamento de \\ Enfermagem Médico \\ Cirúrgica da Escola de \\ Enfermagem da \\ Universidade de São Paulo \\ (EEUSP).rturrini@usp.br \\ 2 Professor Livre Docente \\ do Departamento de \\ Enfermagem Médico \\ Cirúrgica da EEUSP, \\ kugrazia@usp.br, \\ 3 Professor Doutor do \\ Departamento de \\ Enfermagem Médico \\ Cirúrgica da EEUSP. \\ nephron@usp.br \\ 4 Professor Livre Docente \\ do Departamento de \\ Enfermagem Médico \\ Cirúrgica da EEUSP, \\ mtmllf@usp.br
}

\begin{abstract}
RESUMO
Este estudo propôs avaliar o perfil dos alunos e os conhecimentos produzidos no Programa de Pós-Graduação em Enfermagem na Saúde do Adulto (PROESA), por meio de um estudo documental, exploratório e descritivo, desdeacriação do Programa (2000) até setembro de 2004. A demanda dos alunos do PROESA no período analisado foi, predominantemente, de egressos do curso de graduação da EEUSP (46,5\%), sem experiência em iniciação científica, com elevada média de tempo de formado, desempenhando prática assistencial. No período analisado foram concluídas 43 produções (2,4 produções/docente). Dentreas linhas depesquisa, o destaque foi para a linha “Tecnologia na saúde do adulto”. Quanto ao delineamento das pesquisas prevaleceu o não intervencionista e a maioria de trabalhos descritivos. A divulgação dos conhecimentos está sendo ampliada paulatinamente, frente à identificação de queomaior agentedificultadoréolongo período de espera para a concre-

\section{ABSTRACT}

The aim of this study was to evaluate the profile of the students and the knowledge produced in the Adult Health Nursing Graduate Program (PROESA) of the Nursing College of the University of São Paulo(EEUSP). A documental, exploratory and descriptive study from 2000 to 2004 was conducted. Results identified that the students of the PROESA in the period analyzed were graduated at the EEUSP (46.5\%), they had a long time as nursing practitioners and no previous experience in scientific research. It were concluded 43 academic production (2.4 production/ sponsor) and the principal area of investigation was "Technology in adulthealth”. The research designs of most quantitative studies were classified as non-intervention with a high frequency of descriptive studies. The long time to accomplish the publication and the restricted assort of qualified journals in nursing areas were the most important identified factors to limit the publications of the academic production.
\end{abstract} tização das publicações e o pequeno número de periódicos com Qualis Ae B CAPES, preferencialmente internacional, naárea da enfermagem.

\section{DESCRITORES}

Saúde do adulto.

Pesquisa em enfermagem.

Educação de pós-graduação em enfermagem.

\section{KEY WORDS}

Adult health.

Nursing research.

Education, nursing, graduate.

\section{RESUMEN}

La propuesta en este estudio fue evaluar el perfil de los alumnos y los conocimientos producidos en el Programa de Postgrado en Enfermería en Salud del Adulto (PROESA), por medio de un estudio documental, exploratorio y descriptivo, desde la creación del Programa (2000) hasta setiembre del 2004. La demanda de alumnos del PROESA en el período analizado fue, predominantemente, de egresados del curso de pregrado de la EEUSP(46,5\%), sin experiencia en iniciación científica, con elevadopromediodetiempodeegresado, que desempeñaban práctica asistencial. En el período analizado se concluyeron 43 producciones (2,4 producciones/docente). Entre las líneas de investigación,sedestacólade“Tecnología en la salud del adulto”. En cuanto al delineamiento de las investigaciones prevaleció el no intervencionista y la mayoría de trabajos fueron descriptivos. La difusión de los conocimientos está siendo ampliada paulatinamente, frente a la identificación del mayor agente dificultador que es el largo período de espera para la concretización de las publicaciones y el pequeño número de revistas con Cualis A y B CAPES, de preferencia intemacional, en eláreadela enfermeńa.

\section{DESCRIPTORES}

Salud del adulto.

Investigación en enfermería. Educación de postgrado en enfermería. 


\section{PROESA: ASPECTOS HISTÓRICOS}

O Programa de Pós-Graduação Enfermagem na Saúde do Adulto (PROESA), da Escola de Enfermagem da Universidade de São Paulo, foi recomendado pela Coordenação de Aperfeiçoamento de Pessoal de Ensino Superior (CAPES) em julho de 2002, e originou-se da fusão das Áreas de Concentração "Fundamentos de Enfermagem" e "Enfermagem na Saúde do Adulto Institucionalizado”, ambas do Programa de Pós-Graduação em Enfermagem (PPGE) da mesma Instituição de Ensino Superior, que surgiu em 1973. A união dessas duas áreas resultou do reconhecimento de que haveria benefícios de natureza científica, acadêmica e administrativa para a PósGraduação. As duas áreas tinham superposição de corpo docente e, freqüentemente os estudos da área de Fundamentos de Enfermagem, quando clínicos, tinham adultos como sujeitos ${ }^{(1)}$.

O PROESA, apesar de ter sido recomendado pela CAPES somente em julho de 2002, vinha desenvolvendo suas atividades desde janeiro de 2000, com a autorização da Pró-Reitoria de Pós-Graduação da Universidade de São Paulo (USP). Sua vocação principal é capacitar enfermeiros para os processos de produção do conhecimento em tecnologias avançadas do cuidar clínico no âmbito da enfermagem na atenção à saúde do adulto, integrando conhecimentos da ciência da enfermagem aos de outras disciplinas ${ }^{(1)}$.

O PROESA articula três eixos temáticos, a saber: o Cuidar Clínico, o Contexto do Cuidar e a Produção de Ensino Superior. Esses três eixos abrangem cinco linhas de pesquisa e trinta e uma disciplinas. $\mathrm{O}$ eixo temático "Cuidar Clínico" engloba três linhas de pesquisa: "Cuidar do adulto com afecções crônicas não transmissíveis”, "Cuidar do adulto com afeç̧ões agudas e críticas” e "Tecnologia na Saúde do Adulto". O eixo temático "Contexto do Cuidar" consiste em duas linhas de Pesquisas: "Dinâmica de trabalho na saúde do adulto" e "Tecnologia na Saúde do Adulto". Por fim, o eixo "Produção do Ensino Superior" abarca a linha de Pesquisa "Processo ensino-aprendizagem na saúde do adulto". Nessas cinco linhas de pesquisas identificam-se atualmente quarenta projetos em andamento ${ }^{(1)}$.

O corpo docente está constituído por dezoito professores desenvolvendo atividades de ensino, pesquisa e extensão, sendo treze destes classificados como NRD6 ${ }^{(a)}$.

O atual registro (Dezembro de 2004), demonstra o envolvimento de cento e dezoito (118) discentes nas atividades acadêmicas do PROESA, sendo vinte e seis alunos de graduação, cinqüenta e seis de Mestrado e trinta e seis de Doutorado.
Neste cenário, este estudo teve por objetivo responder a duas questões norteadoras: Qual é o perfil dos alunos que procuraram o PROESA até 2004? Como está caracterizada a produção de conhecimentos do Programa até 2004?

\section{MATERIALE MÉTODO}

Trata-se de um estudo documental, exploratório e descritivo, de registros referentes ao período de 2000 a 2004. Como fonte de dados foram utilizados as fichas de inscrição e cadastros dos alunos na pós-graduação, data da defesa das dissertações ou teses e os respectivos resumos da produção científica. Algumas informações foram obtidas diretamente com os orientadores do PROESA.

A caracterização dos alunos pautou-se nas seguintes variáveis: local de moradia, tempo de formado, escola em que se graduou, campo de atuação no momento da primeira matrícula e ao final da pós-graduação, participação no Programa de Aperfeiçoamento de Ensino (PAE) e a obtenção de bolsa.

O estudo do conhecimento produzido reuniu os seguintes dados sobre os trabalhos de conclusão (dissertações ou teses): linha de pesquisa, tipo de pesquisa, delineamento metodológico, tema central e divulgação do conhecimento.

Os dados foram analisados por freqüências absolutas e relativas; para as variáveis quantitativas contínuas foram calculadas média, mediana e desvio padrão.

\section{RESULTADOSE DISCUSSÃO}

\section{Perfil dos ingressantes}

No período do estudo, 2000 a 2004, 43 alunos concluíram a pós-graduação (38 mestres e cinco doutores). Do total, 20 (46,5\%) haviam concluído o curso de graduação na USP, sendo 18 (41,9\%) deles na própria EEUSP; quatro $(9,4 \%)$ na Universidade Federal de São Paulo, dez (23,3\%) em outras escolas privadas do Estado de São Paulo, e nove (20,8\%) em escolas de outros Estados. Somente dois alunos não eram residentes de São Paulo.

Entre os alunos incluídos no estudo, apenas dois $(4,7 \%)$ tinham registro de participação em programa de iniciação científica durante a graduação. Esse achado reforça a necessidade de adoção de medidas que fortaleçam as iniciativas de inserção precoce dos graduandos em pesquisa. Fica claro que a incorporação de monografias de conclusão de curso na graduação, outra forma de inserir o aluno em pesquisa é recente, e a EEUSP foi uma das pioneiras nesta conduta, há sete anos.
Perfil dos alunos e conhecimentos produzidos no Programa de Pós-Graduação de Enfermagem na Saúde do Adulto (a) NRD6 docente com regime de trabalho mínimo de 30 horas em Instituição de Ensino Superior com dedicação ao Programa superior a $30 \%$ da carga horária contratada, por mais de 9 meses no Ano-Base.

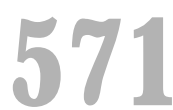

Rev Esc Enferm USP 2005; 39(Esp.):570-9. 
Ruth Natália Teresa Turrini Kazuko Uchikawa Graziano Maria de Fátima F. Vattimo Diná de A. L. M. da Cruz
O tempo médio de formado dos alunos que ingressaram no mestrado foi de $9,9 \pm 5,9$ anos, mediana de 10,5 anos (variação de 0 a 22 anos) e do doutorado de 15,2 $\pm 3,3$ anos, mediana de 15 anos (variação de 11 a 20 anos). A elevada média de tempo de formados mostra um panorama desfavorável na perspectiva de formação de jovens doutores. Por outro lado, construir o conhecimento com alunos de maior experiência na prática profissional traz benefícios pelas reflexões e discussões que podem ser realizadas com aproveitamento para ambas as partes, professor e aluno.

Quanto à atuação profissional do corpo discente observou-se que no mestrado, 32 (84,2\%) alunos desempenhavam atividades assistenciais, um (2,6\%) acumulava atividades no ensino superior e na assistência, e cinco (13,2\%) somente no ensino superior. O inverso verificou-se no doutorado: três $(60,0 \%)$ eram docentes e dois $(40,0 \%)$ assistenciais. Neste estudo não foram exploradas as expectativas dos alunos com a obtenção dos títulos. No entanto, é possível admitir que o mestrado seja visto como estratégia válida para continuar o processo formal de educação ou como um estágio de preparação como pesquisador, o que explicaria a freqüência observada de alunos de mestrado desempenhando atividades assistenciais. Certamente, preparar-se para um futuro ingresso na área do ensino superior poderia também explicar esse resultado.

Quanto ao doutorado, apesar do pequeno número de alunos no período estudado, observa-se que a maior proporção é de enfermeiros já vinculados com a docência, que é um espaço de maior incentivo para a formação de pesquisador. Ainda que tênue, observa-se tendência de o doutor em enfermagem ganhar espaço nas instituições assistenciais, como pesquisador, coordenando núcleos de investigação e oferecendo assessoria metodológica para o desenvolvimento de pesquisas.
Com relação às bolsas de Pós-Graduação, foi observado que a maioria dos alunos não a possuía e o motivo principal era terem vínculo empregatício: 31 (81,6\%) alunos do mestrado e quatro $(80,0 \%)$ alunos do doutorado. Dentre os alunos do mestrado com bolsa, dois (5,3\%) tinham bolsa do Conselho Nacional de Pesquisa (CNPq), três (7,9\%) da CAPES e um (2,6\%) da Fundação de Amparo a Pesquisa do Estado de São Paulo (FAPESP). Somente um (20,0\%) aluno do doutorado tinha bolsa que era proveniente da CAPES.

A oferta de bolsa pode ser um ganho para o aluno e para o programa. A existência de bolsas no Programa de Pós-Graduação permite que o aluno participe mais intensamente das atividades acadêmicas, garantindo um leque mais amplo de experiências acadêmicas, favorecendo maior produtividade e cumprimento do tempo de titulação.

A elevação do número de bolsas émeta do PROESA para a qual têm sido estabelecidas políticas de seleção de alunos que demonstrem interesse e possibilidade de se dedicar somente à pós-graduação.

O Programa de Apoio ao Ensino (PAE) foi instituído na Universidade de São Paulo em 1992. Esse programa tem por objetivo dar subsídios aos alunos de mestrado e doutorado para exercitarem a atividade docente de ensino superior e representa uma estratégia institucional inovadora na complemen-tação da formação do aluno. No período estudado, apenas três $(9,3 \%)$ alunos do mestrado, participaram do PAE.

\section{PROESA: PRODUÇÃO CIENTÍFICA}

Desde sua criação o PROESA produziu 43 investigações no âmbito da pós-graduação, sendo 38 dissertações de mestrado e cinco teses de doutorado. Esse desempenho confere um fluxo de 8,6 investigações por ano no período analisado (Gráfico 1).

Gráfico 1 - Número de investigações concluídas por ano no PROESA-EEUSP. São Paulo, 2000-2004.

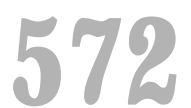

Rev Esc Enferm USP 2005; 39(Esp.):570-9.

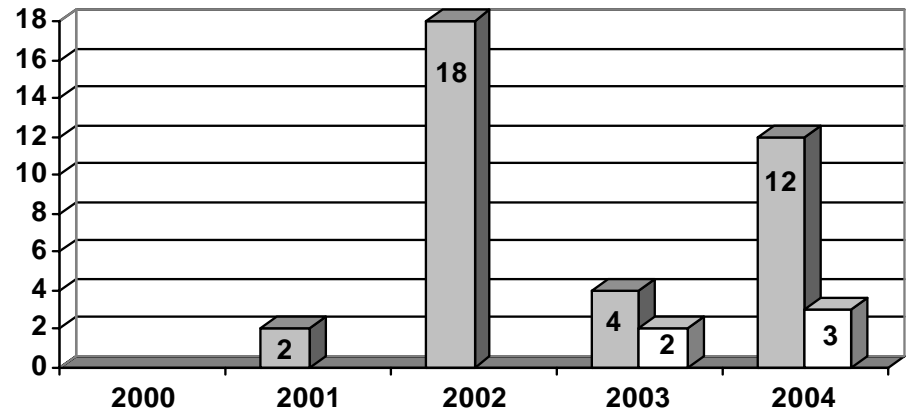

$\square$ Mestrado

$\square$ Doutorado 
A produção de conhecimentos do PROESA iniciou-se efetivamente em 2001 com uma média de 10,8 produções por ano para o período de 2000 a 2004. Nesse período os docentes do PROESA ainda tinham orientações nos outros programas de pósgraduação da EEUSP, o que contribuiu para a irregularidade de produções no PROESA. A dedicação exclusiva dos docentes ao PROESA permitirá que o número de conclusões por ano seja mais uniforme.

O tempo médio de titulação do mestrado no período foi de 30,3 $\pm 3,1$ meses, com uma mediana de 31 meses (variação de 21 a 38 meses). Tem havido investimentos numa política para reduzir o tempo de titulação. Com a aprovação da nova Norma da PósGraduação do PROESA, é exigido do aluno o depósito das dissertações até 27 meses da sua primeira matrícula e com isso espera-se garantir a defesa até os 30 meses. Para o doutorado, o tempo médio de titulação foi de 49,2 $\pm 3,3$ meses, mediana de 50 meses (variação de 46 a 54 meses). No caso do doutorado, esse período foi superior a tempo preconizado (48 meses) em decorrência da existência de um caso de doutorado direto que prevê um tempo de titulação de 54 meses. Portanto, todos os alunos do doutorado cumpriram os prazos estabelecidos. O doutorado direto na EEUSP é aceito em casos de candidatos que comprovem expressiva produção científica na área temática pretendida, engajamento em grupos de pesquisa e experiência na orientação de trabalhos científicos.

Na Tabela 1 está apresentada a distribuição das investigações do PROESA por linha de pesquisa.

Tabela 1 - Distribuição de investigações concluídas no PROESA-EEUSP por linhas de pesquisa São Paulo, 2000-2004.

\begin{tabular}{lrr}
\hline \multicolumn{1}{c}{ Linha de Pesquisa } & N & $\%$ \\
\hline Tecnologias do cuidar na saúde do adulto & 19 & 44,2 \\
O cuidar na saúde do adulto do paciente com doenças agudas ou críticas & 9 & 20,9 \\
Dinâmica de trabalho & 9 & 20,9 \\
O cuidar na saúde do adulto do paciente com doenças crônicas & 3 & 7,0 \\
Ensino-aprendizagem & 3 & 7,0 \\
\hline Total & 43 & \multirow{2}{*}{100,0} \\
\hline
\end{tabular}

A distribuição apresentada na Tabela 1 mostra que houve predominância de investigações voltadas às tecnologias do cuidar na saúde do adulto (44,2\%). O que é coerente com a missão do PROESA de produzir conhecimentos em tecnologias avançadas do cuidar clínico. Adicionalmente, os estudos na área de tecnologias do cuidar subsidiam a assistência tanto em situações agudas quanto crônicas, o processo de trabalho e o ensino-aprendizagem.

Com referência às sociedades já industrializadas ou a caminho da industrialização o termo Tecnologia designa a totalidade ou um setor organizador do conjunto de conhecimentos sobre: a) princípios e descobertas científicas e b) processos industriais existentes ou antigos, fontes de poder e matérias primas e métodos de transmissão e comunicação considerados importantes para a produção ou o aperfeiçoamento de mercadorias e serviços. Tecnologia pode ser entendida como a ação que o indivíduo desempenha sobre um objeto com ou sem ajuda de instrumentos ou dispositivos mecânicos, a fim de fazer alguma mudança naquele objeto. $\mathrm{O}$ objeto ou a matéria prima podem ser: o ser vivo, humano ou outra forma, um símbolo ou um objeto inanimado. Tecnologia é também processo, ato ou ação $0^{(2)}$.
A análise da produção na linha de Tecnologia (Anexos I) permite observar um foco preferencial na criação, adaptação ou validação de instrumentos de medida (7- 36,8 \%). Essa tendência reitera a busca por modelos objetivos de dimensionamento das necessidades do cuidar do paciente, resultados do cuidar, cargas de trabalho e custos na assistência à saúde, ou seja, estratégias consistentes para quantificar o cuidado na saúde do adulto nas suas diversas interfaces. Ainda em relação à Tabela 1, as linhas "Ensino-aprendizagem" e "O cuidar na saúde do adulto do paciente com doenças crônicas" apresentaram a menor produção $(7,0 \%)$ no período, fato atribuído ao menor número de docentes do programa voltados para esse tema.

Partindo do princípio de que para um cuidado de qualidade é preciso desenvolver tecnologias foi considerado aceitável o destaque da linha de investigação "Tecnologia na saúde do adulto" para o PROESA nestes cinco anos analisados.

Esse panorama de produção distribuído nas cinco linhas de pesquisa do PROESA será objeto de avaliação contínua pelos docentes do programa para que se verifique a necessidade de fortalecimento das duas linhas que apresentaram menor produção ou de recomposição das linhas e projetos de pesquisa (Anexos II-V).
Perfil dos alunos e conhecimentos produzidos no Programa de Pós-Graduação de Enfermagem na Saúde do Adulto 
Ruth Natália Teresa Turrini Kazuko Uchikawa Graziano Maria de Fátima F. Vattimo Diná de A. L. M. da Cruz
Das sete disciplinas obrigatórias do curso de graduação oferecidas pelo Departamento Enfermagem Médico-Cirúgica, onde estão alocados todos os docentes do PROESA, duas delas são direcionadas para os pacientes críticos, e as demais contemplam tanto o paciente crítico quanto o crônico. Esse fato também pode justificar a predominância da produção de conhecimentos na linha do cuidado ao paciente crítico sobre a de crônicos. A classificação da produção científica nas linhas de pesquisa foi baseada no título atribuído com seu respectivo resumo. A classificação de uma produção científica na linha agudo ou crônico nem sempre foi uma tarefa fácil neste estudo. Por exemplo, na investigação que enfoca a agudização de uma doença crônica, a decisão foi classificá-la na linha de agudos.

A atividade didática dos docentes orientadores do PROESA na graduação deve estar em consonância com a área temática das investigações do docente. Essa conduta está de acordo com o pressuposto do Ministério da Educação e Cultura (MEC), de indissociabilidade do ensino, pesquisa e extensão. O PROESA possui mais docentes que desenvolvem projetos relacionados a pacientes em situações agudas do que a pacientes em situações crônicas.
A inexistência de uma classificação con-sensual sobre delineamentos de pesquisa dificultou a tarefa de categorizar as pesquisas neste estudo. Diferentes autores de áreas distintas propõem classificações particulares. Para este trabalho adotamos a da Polit e Hungler ${ }^{(3)}$, com pequenas adaptações na classificação descritiva subdividindo-a em simples, comparativa/correlacional e longitudinal. Assim sendo, os conhecimentos produzidos pelo PROESA foram agrupados, primeiramente, em dois grandes grupos: pesquisas com intervenção e sem intervenção. Os com intervenção foram subdivididos em pesquisa (1) experimental em laboratório, (2) experimental em campo, (3) quase experimento quando a pesquisa não trabalhou com grupo controle. Os sem intervenção foram subdivididos em pesquisa (4) descritiva simples, (5) descritiva expost-facto, (6) descritiva longitudinal, (7) descritiva comparativo/correlacional, (8) metodológica, (9) descritiva com abordagem qualitativa.

A Tabela 2 apresenta os dados referentes às investigações realizadas pelo PROESA no período analisado. Na mesma tabela, os estudos estão apresentados conforme o delineamento de pesquisa instituído, com intervenção ou sem intervenção, distribuídos entre as linhas de pesquisa.

Tabela 2 - Distribuição de investigações do PROESA-EEUSP segundo linhas de pesquisa e método São Paulo, 2000-2004.

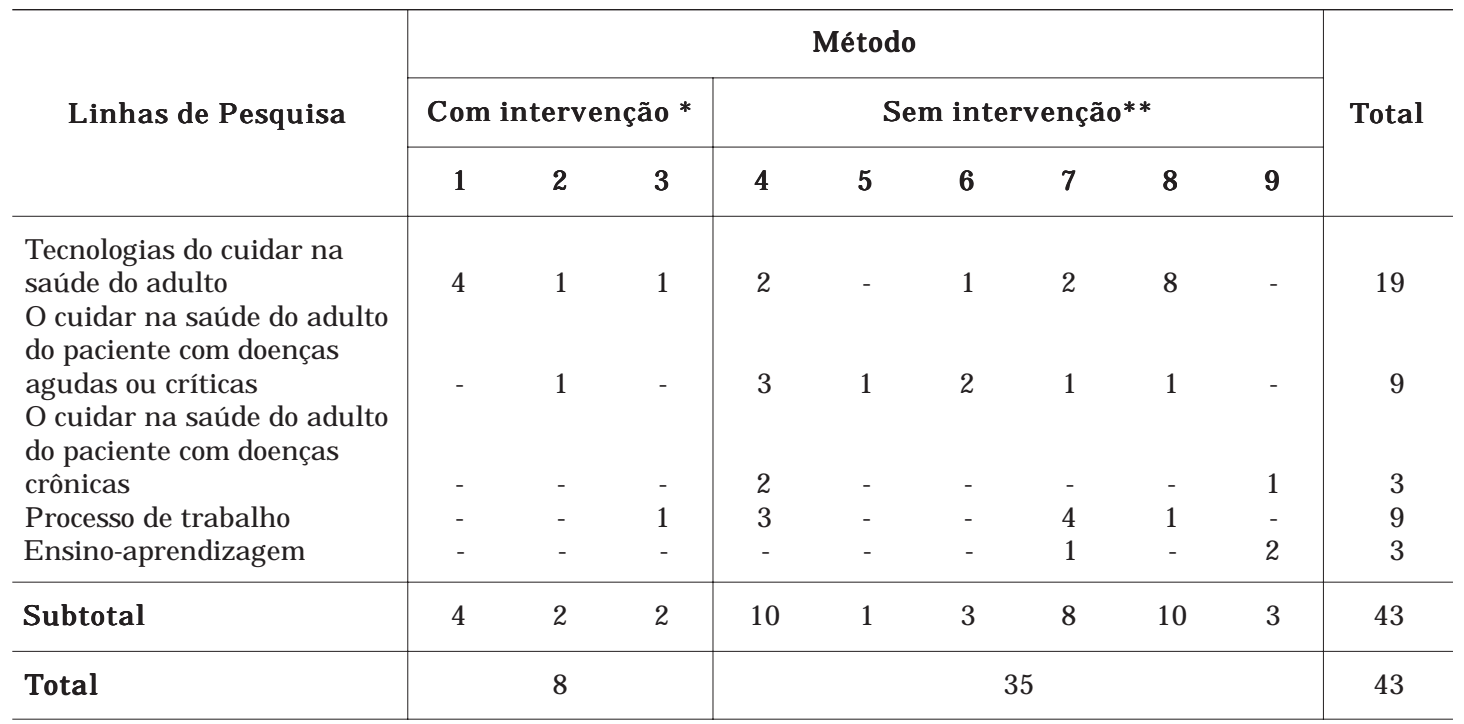

*Com intervenção: $1=$ experimental em laboratório; $2=$ experimental em campo; $3=$ quase experimento

** Sem intervenção: $4=$ descritiva simples; $5=$ descritiva post-facto; $6=$ descritiva longitudinal; $7=$ descritiva comparativa/correlacional; $8=$ metodológica; $9=$ descritiva com abordagem qualitativa

A maioria dos estudos $(72,9 \%)$ realizados foi classificada como pesquisa "sem intervenção", com maior freqüência de pesquisas metodológicas (28,6\%), descritiva simples (28,6\%) e descritiva comparativa/correlacional (22,8\%).
Um conhecimento é gerado na sequiência de três níveis: o primeiro nível de geração de conhecimentos de um determinado assunto investigado é a exploração e descrição do fenômeno para conhecê$\mathrm{lo}^{(4)}$. Nesse tipo de investigação, a estatística utili-
Rev Esc Enferm USP 2005; 39(Esp.):570-9. 
zada é a estatística descritiva e não inferencial. A partir do conhecimento suficiente do fenômeno, vem o segundo nível de geração de conhecimento representado pelos estudos comparativos/correlacionais. No terceiro nível, o delineamento prevê uma intervenção a ser testada pelo pesquisador, o que caracteriza os estudos experimentais. No segundo e terceiro níveis são utilizadas estatísticas inferenciais.

Observa-se entre os pesquisadores e também nas políticas dos órgãos de fomento à pesquisa maior valorização dos estudos com intervenção. O conhecimento produzido com estudos desse tipo tem, potencialmente, maior impacto e aplicabilidade. No entanto, quando um fenômeno de investigação é pouco explorado, o delineamento experimental torna-se inadequado, daí a importância dos estudos iniciais descritivos exploratórios. Os estudos qualitativos e quantitativos que descrevem e exploram fenômenos pouco conhecidos, pouco delimitados, trazem grandes contribuições para a enfermagem na saúde do adulto.

Os estudos metodológicos se caracterizaram pelo desenvolvimento ou refinamento de instrumentos de medidas de variáveis qualitativas como, por exemplo, estudos de adaptação e validação de escalas de qualidade de vida.

A Tabela 3 apresenta as produções do PROESA segundo os as variáveis ou conceitos centrais investigados e método.

Tabela 3 - Distribuição de investigações do PROESA segundo variáveis medidas ou conceitos centrais estudados - São Paulo, 2000-2004.

\begin{tabular}{|c|c|c|c|c|c|c|c|c|c|c|}
\hline \multirow{3}{*}{ Variáveis centrais } & \multicolumn{9}{|c|}{ Método } & \multirow{3}{*}{ Total } \\
\hline & \multicolumn{3}{|c|}{$\underset{\text { intervenção }}{\text { Com }}$} & \multicolumn{6}{|c|}{ Sem intervenção** } & \\
\hline & 1 & 2 & 3 & 4 & 5 & 6 & 7 & 8 & 9 & \\
\hline Avaliação da função renal & 1 & - & - & 1 & - & 1 & - & - & - & 3 \\
\hline Avaliação da função intestinal & - & - & - & - & - & - & - & 1 & - & 1 \\
\hline Avaliação da gravidade de pacientes em UTI & - & - & - & - & - & 1 & 1 & - & - & 2 \\
\hline Avaliação da fadiga & - & - & - & - & - & - & - & 1 & - & 1 \\
\hline Avaliação da dor & - & - & - & - & - & - & - & 1 & - & 1 \\
\hline Complicações pós operatórias & - & - & - & 1 & 1 & - & - & - & - & 2 \\
\hline Ansiedade/stress/coping & - & 1 & - & - & - & - & 3 & - & - & 4 \\
\hline Infecção & - & - & - & - & - & - & 1 & - & - & 1 \\
\hline Intervenção vibracional & - & - & 1 & - & - & - & - & - & - & 1 \\
\hline Sistematização da assistência de enfermagem & - & 2 & - & 2 & - & - & - & - & - & 4 \\
\hline Cuidador do paciente no domicílio & - & - & - & 1 & - & 1 & - & - & - & 2 \\
\hline Emoção/sentimentos & - & - & - & - & - & - & 1 & - & 1 & 2 \\
\hline Crenças & - & - & - & 1 & - & - & - & - & - & 1 \\
\hline Comunicação & - & - & - & 1 & - & - & - & - & 1 & 2 \\
\hline Suspensão de cirurgias & - & - & - & - & - & - & - & - & - & 1 \\
\hline Custos & - & - & - & - & - & - & - & 1 & - & 1 \\
\hline Reuso de artigo único & - & - & - & - & - & - & - & 1 & - & 1 \\
\hline Limpeza e desinfecção & 1 & - & - & - & - & - & - & - & - & 1 \\
\hline Antissépticos & 1 & - & - & - & - & - & - & 1 & - & 2 \\
\hline Máscara cirúrgica & 1 & - & - & - & - & - & - & - & - & 1 \\
\hline Programa de controle de infecção hospitalar & - & - & - & 1 & - & - & - & - & - & 1 \\
\hline Qualidade de vida & - & - & - & - & - & - & 1 & 3 & - & 4 \\
\hline Carga de trabalho & - & - & - & - & - & - & - & 1 & - & 1 \\
\hline SUS & - & - & - & 1 & - & - & - & - & - & 1 \\
\hline Competências & - & - & 1 & - & - & - & - & - & 1 & 2 \\
\hline Total & 4 & 3 & 2 & 9 & 1 & 3 & 8 & 10 & 3 & 43 \\
\hline
\end{tabular}

*Com intervenção: $1=$ experimental em laboratório; $2=$ experimental em campo; $3=$ quase experimento

**Sem intervenção: $4=$ descritiva simples; $5=$ descritiva post-facto; $6=$ descritiva longitudinal; $7=$ descritiva comparativa/correlacional; $8=$ metodológica; $9=$ descritiva com abordagem qualitativa

Perfil dos alunos e conhecimentos produzidos no Programa de Pós-Graduação de Enfermagem na Saúde do Adulto 
Ruth Natália Teresa Turrini Kazuko Uchikawa Graziano Maria de Fátima F. Vattimo Diná de A. L. M. da Cruz
Nos dados apresentados na Tabela 3 observase uma diversidade de variáveis ou conceitos centrais que se distribuem entre os desenhos metodológicos de maneira não sistemática, à exceção dos estudos referentes à Qualidade de Vida em que foram empregados preferencialmente os métodos sem intervenção do tipo quantitativo simples.

Até a época da preparação deste artigo, somente $11(25,6 \%)$ das dissertações ou teses tinham sido publicadas na íntegra em periódicos, e os demais estavam em fase de julgamento ou elaboração. $\mathrm{O}$ tempo médio para publicação de um artigo na área da enfermagem tem levado cerca de 12 a 24 meses. $\mathrm{O}$ PROESA tem incentivado a publicação de artigos em periódicos durante o desenvolvimento da pósgraduação e não só ao término do curso.

Apesar da baixa proporção de estudos divulgados na forma de artigos em periódicos até a época da preparação deste artigo, todos os estudos já tinham sido apresentados em eventos científicos. Também a divulgação dos conhecimentos em livros e em capítulos de livros tem sido uma alternativa expressiva de publicação.

Os resumos das dissertações e teses foram avaliados quanto à clareza e presença dos itens: objetivos, método e principais resultados numa escala de 1 a 5 (5=melhor pontuação). A média de pontuação alcançada foi de $4,3 \pm 1$. O resumo dos estudos é o primeiro veículo de divulgação dos conhecimentos produzidos e um convite para a busca do trabalho na íntegra; daí a importância no cuidado da sua elaboração.

\section{CONCLUSÃO}

O PROESA é um Programa de Pós-Graduação jovem - quando é considerado o tempo da sua exis- tência - porém, constituído de orientadores com longa experiência em orientação e produção de estudos na pós-graduação. No período analisado foram produzidas 43 investigações (38 dissertações e cinco teses) o que significa 2,4 produções para cada um dos 18 docentes que compõem o PROESA. Dentre as linhas de pesquisa, o destaque foi para a linha “Tecnologia na saúde do adulto”. Predominaram estudos sem intervenção do tipo descritivo. $\mathrm{O}$ aspecto que necessita de maior empenho para superar as dificuldades é o da divulgação dos conhecimentos produzidos nas dissertações e teses.

A demanda dos alunos do PROESA no período analisado foi, predominantemente de egressos da EEUSP (41,9\%), sem experiência em iniciação científica com elevada média de tempo de formado desempenhando prática assistencial e, o vínculo empregatício tem sido limitante para a obtenção de bolsas e participação do PAE.

Essa síntese sugere alguns pontos que, provavelmente, influenciam-se mutuamente. O tempo de graduação elevado pode estar associado à falta de iniciação científica e também à menor predisposição para dedicação exclusiva ao curso, o que impede que o aluno seja bolsista e que tenha participação mais intensa nas atividades acadêmicas. O incentivo atual à iniciação científica é benéfico, mas ainda faltam estratégias que incentivem enfermeiros jovens a ingressarem nos programas de pósgraduação com dedicação integral.

Este estudo apresentou resultados sobre algumas características de um programa de pós-graduação nos seus primeiros 4 anos de funcionamento. Pode servir de referência para outras análises semelhantes e também para o aprofundamento de aspectos aqui levantados.

\section{REFERÊNCIAS}

(1) Pimenta CAM, Cruz DALM, Padilha KG. Programa de Pós-Graduação em Enfermagem na Saúde do Adulto da Escola de Enfermagem da USP. Rev Esc Enferm USP 2004; 38(4):458-66.

(2) Perrow C. A framework for the comparative analysis of organization. Am Soc 1967; 32(2):194-208.
(3) Polit DF, Hungler, BP. Nursing research: principles and methods. $5^{\text {th }}$ Baltimore: Lippincott Williams \& Wilkins; 1995.

(4) Brink PJ, Wood MJ. Basic steps in planning nursing research - front question to proposal. $3^{\text {th }}$ ed. Boston: Jones \& Barlett; 1988. 


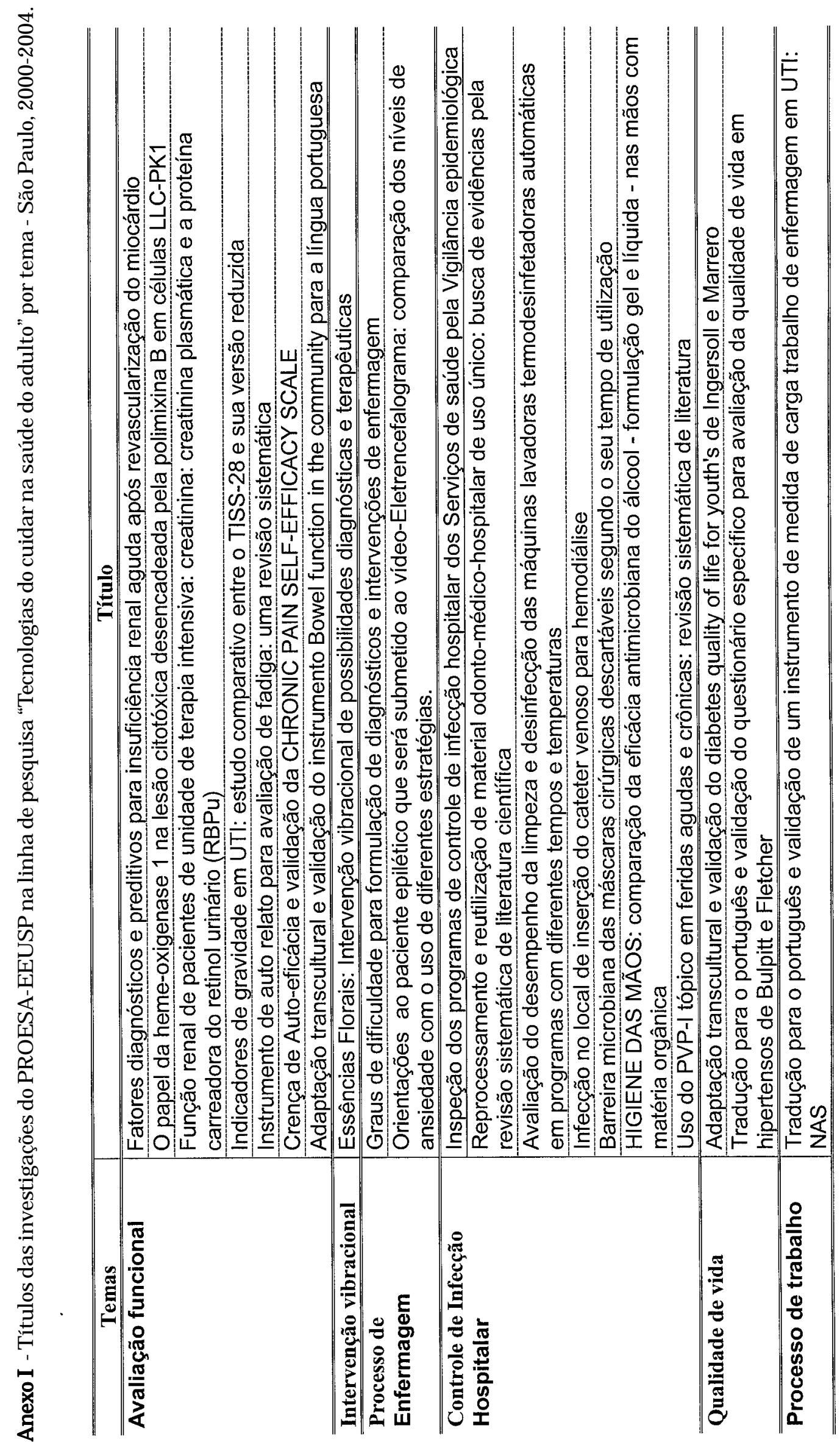

Perfil dos alunos e

conhecimentos

produzidos no Programa

de Pós-Graduação

de Enfermagem na

Saúde do Adulto 

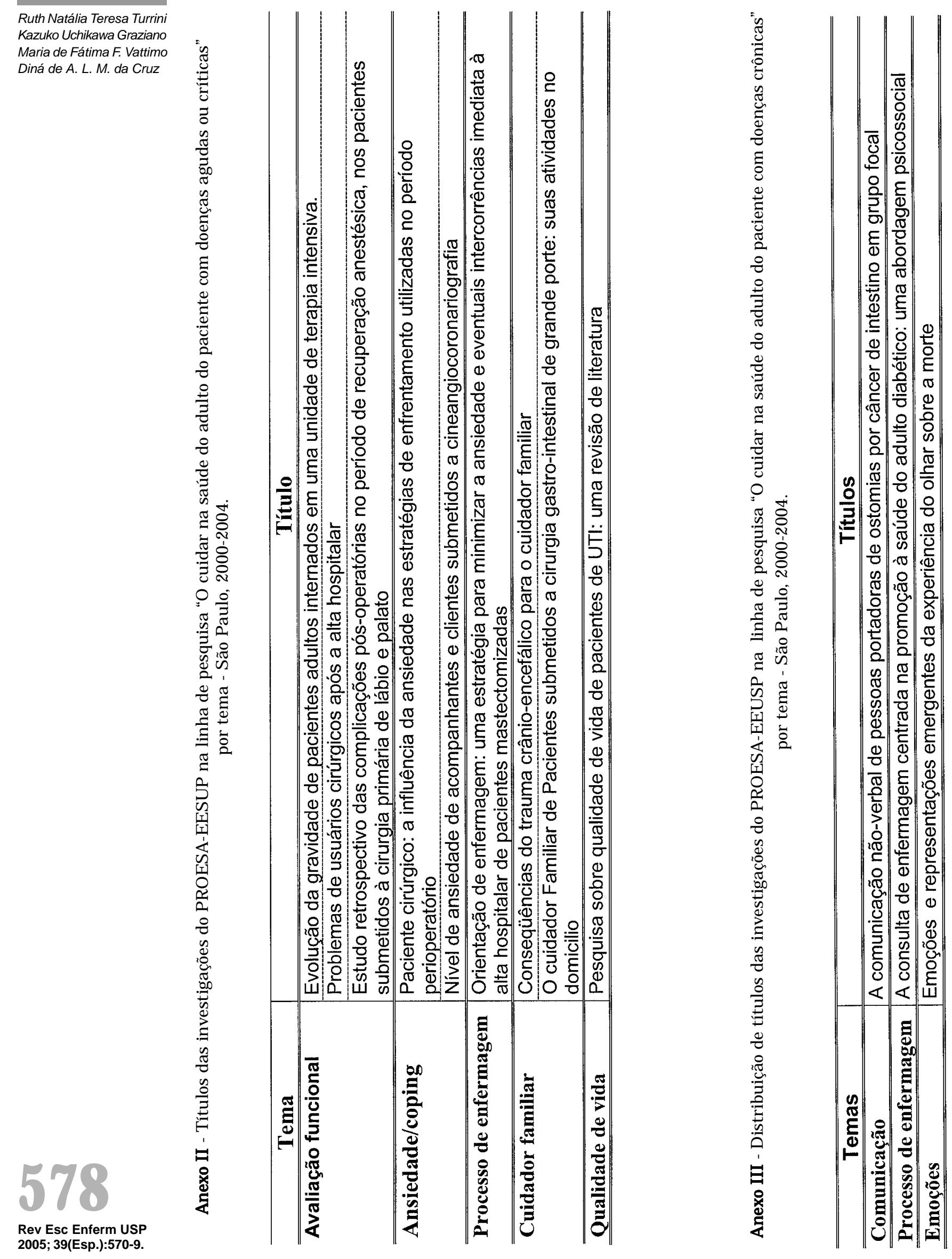


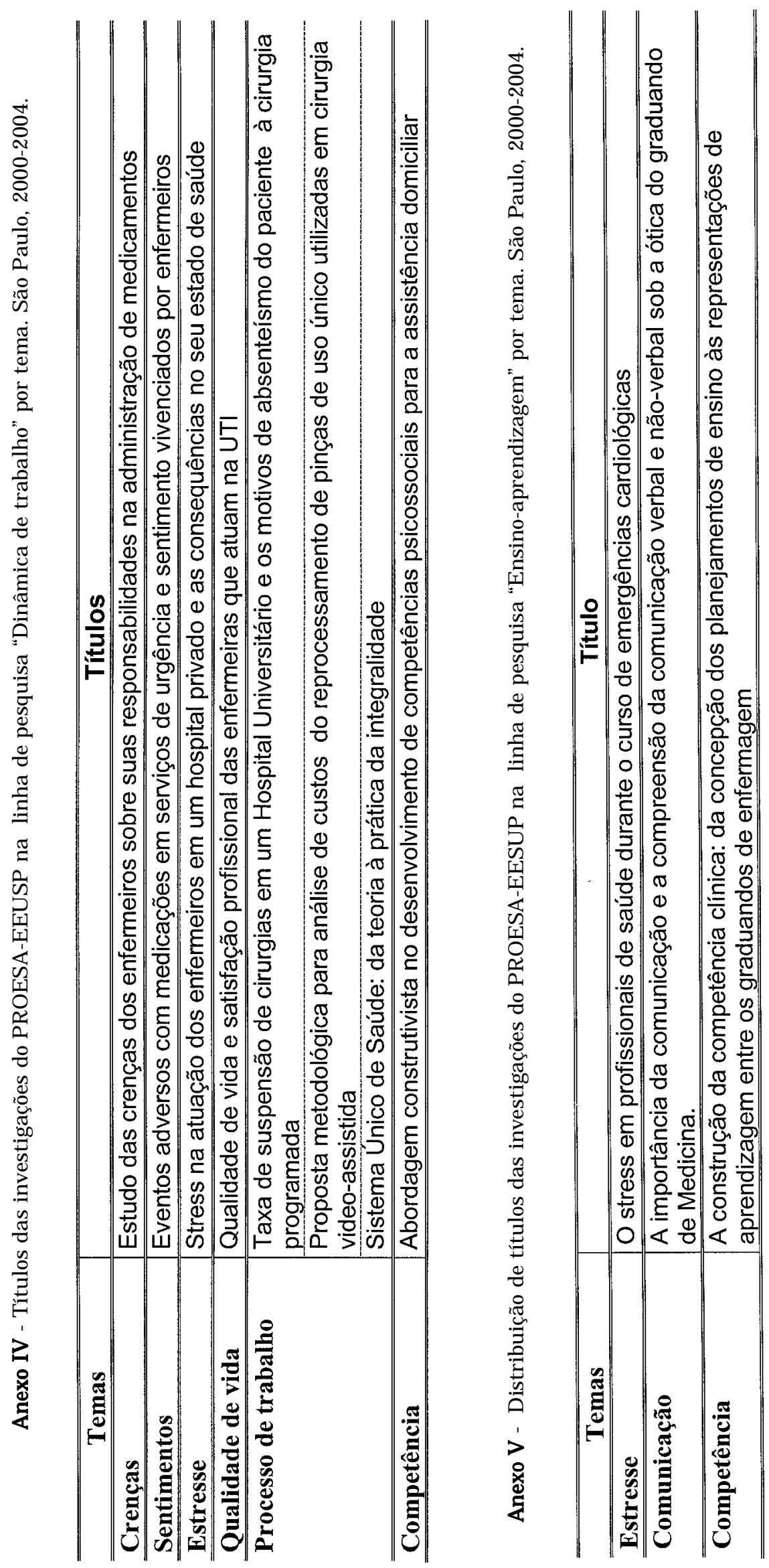

Perfil dos alunos

conhecimentos

produzidos no Programa

de Pós-Graduação

de Enfermagem na

Saúde do Adulto

Correspondência:

Ruth Natália T. Turrini

Av. Dr. Enéas de C.

Aguiar, 419 - Cerqueira

César - São Paulo

05403-000 - SP 